\title{
Fabrication of Transparent and Conductive Microdevices
}

\author{
Szabolcs BEKE ${ }^{1,2 *}$, László KÖRÖSI ${ }^{3}$,Koji SUGIOKA ${ }^{1}$, Katsumi MIDORIKAWA ${ }^{1}$ \\ ${ }^{1}$ Advanced Science Institute, RIKEN, 2-1 Hirosawa, Wako, Saitama 351-0198, Japan \\ ${ }^{2}$ Department of Nanophysics, Italian Institute of Technology, via Morego 30, 16163 Genova, Italy \\ ${ }^{3}$ Supramolecular and Nanostructured Materials Research Group of the Hungarian Academy of Sci- \\ ences, University of Szeged, Dóm tér 8, H-6720 Szeged, Hungary
}

*beke@scientist.com

\begin{abstract}
Microsystems and microdevices can shrink a whole laboratory into a single chip. Due to its small size, such systems are cost effective, as they require small sample sizes and analysis time. Since the silicon-based microchip cannot provide optical transparency, there is a rising interest towards glassbased microchips and their possible applications where optical transparency during the analysis procedure is of high importance. Our objective is to fabricate microdevices and sensors using laser direct writing and thin film deposition techniques. Applying these two methods to transparent materials will open new avenues in microdevice production by enabling the realization of transparent and conductive platforms.

DOI:10.2961/jlmn.2012.01.0005
\end{abstract}

Keywords: Photosensitive glass, ITO, 3D fabrication, femtosecond laser, MEMS, gas sensor, electroluminescent cell

\section{Introduction}

Realizing microdevices on transparent and conductive platforms is of particular benefit to fields where optical transparency, used in spectroscopical/chemical analysis, is crucial.

Microdevices [e.g., lab-on-a-chips (LOC), microelectromechanical systems (MEMS), micro total analysis systems ( $\mu$ TAS)] can shrink a whole laboratory into a single chip that would be utilized in many application areas. To monitor chemical reactions, make biochemical- and clinical analysis, and produce microreactors on a transparent platform are challenging and promising goals. Due to the small size and complexity, such systems provide sensitive performance that is cost effective to manufacture [1]. Nowadays, given that the silicon-based platforms cannot provide optical transparency in the visible spectral range, there is a rising interest towards glass-based devices and its possible applications. There is an urgent need to incorporate conductive but still transparent functions into the microdevices without degrading the transparent feature of the glass. Further, the incorporation of electric functionality into photosensitive glass makes the glass chip an attractive environment also for a wide variety of MEMS applications. The realization of transparent electrodes and microheaters allows not only the regular optical observations and analysis, but also the in-situ optical observations and analysis whilst altering the electric field or/and temperature. For example, such functional systems are beneficial for dynamic observation of living microorganisms and cells.

In this paper, we present a technique to incorporate conductive, but still transparent functions into glass chips by means of fs laser direct writing combined with a subsequent ITO thin film preparation in the channel by a sol-gel process. This idea takes advantage of the high precision of fs laser microfabrication and the cost effectiveness and high adaptability of the sol-gel process.

\subsection{Femtosecond laser processing}

In the past years, femtosecond (fs) laser microfabrication has demonstrated its great potential in materials microprocessing and microdevice manufacturing due to the high peak intensities in extremely short pulse durations allowing the local modification of transparent materials through multiphoton absorption. The most advantageous aspect of this method is the capability of tailoring threedimensional (3D) fluidic, mechanical and optical microcomponents (such as microfluidics, optical waveguides, couplers and splitters, Bragg gratings, Fresnel zone plates, reservoirs, mirrors, lenses, valves, pumps) [2-13]. Our technique of fs laser processing of photosensitive glass and other materials is presented in detail in refs. $[14,15]$.

\subsection{Materials used}

Commercially available photosensitive glass (under the trade name of Foturan ${ }^{\circledR}$ ) is used which combines the unique glass properties (e.g., transparency, hardness, chemical and thermal resistance, etc.), and the possibility of achieving very fine structures with tight tolerances and high aspect ratios (hole depth/hole width) made by fs laser irradiation. This glass is an excellent material to embed hollow microchannels and other complicated 3D structures 
using fs laser direct writing. Foturan glass is comprised of lithium aluminosilicate glass doped with trace amounts of silver and cerium. The cerium $\left(\mathrm{Ce}^{3+}\right)$ ion plays an important role as a photosensitizer, releasing an electron by the exposure of UV light and becoming $\mathrm{Ce}^{4+}$. Some of the silver ions are reduced by the generated free electrons and silver atoms are created. Another asset of the Foturan glass is that it can withstand the $550{ }^{\circ} \mathrm{C}$ calcination temperature of the xerogel films. (After the starting sol is dropped on the Foturan glass, it dries and becomes a xerogel.) This high temperature is necessary for the ITO film crystallization and conductivity.

Among a variety of glass materials, the photosensitive glass is one of the most promising one for these applications and its utilization has been continuously developing [9-13]. Lately, there were many studies on the possible applications of this technique in the field of microfluidics, LOC- and $\mu$ TAS devices, however, there is still a high demand to further improve the complexity and production cost of these microdevices as well as their functionalities.

The other material we use is one of the most-widely used transparent conducting oxides, indium tin oxide (Sndoped $\mathrm{In}_{2} \mathrm{O}_{3}$, ITO) which is an n-type semiconductor with an optical band gap between $3.5-4.3 \mathrm{eV}$. It provides high electrical conductivity and transparency in the visible and near IR (infrared) wavelengths. Hence, it is widely used as transparent electrodes for the fabrication of liquid crystal displays (LCDs), organic light emitting diode displays (OLEDs) $[16,17]$, photovoltaic devices $[18,19]$, and other optical applications [20-22]. ITO thin films can be prepared by various methods, such as sputtering [23], evaporation [24,25], spray pyrolysis [26], and screen printing [27]. However, recently, the sol-gel process became widespread due to its simplicity and low cost [28-34].

\section{Methods}

The idea to realize transparent and conductive functions incorporated into the glass chips relies on the combination of two materials and methods (Fig. 1.). Fabrication of hollow 3D microstructures inside the photosensitive glass consists of a four-step process. The detailed experimental outline to embed conductive ITO thin films in the glass chip is presented in Fig. 1:

(a) 3D photomodified structures are written inside the photosensitive glass using fs laser direct writing. At this stage, silver atoms are precipitated, but no visible change occurs. After the exposure of the fs laser,

(b) the glass chip is annealed in a programmed furnace. The temperature is first ramped up to $500{ }^{\circ} \mathrm{C}$ at a rate of 5 ${ }^{\circ} \mathrm{C} / \mathrm{min}$ and held at this temperature for 1 hour. It is then raised to $605{ }^{\circ} \mathrm{C}$ at a rate of $3{ }^{\circ} \mathrm{C} / \mathrm{min}$ and held there for another hour. Upon this treatment, the laser-exposed region become crystallized lithium metasilicate and shows a brown color. After this step,

(c) the glass is soaked in a solution of $10 \%$ hydrofluoric (HF) acid diluted with water in ultrasonic bath. During this process, the formed lithium metasilicate is preferentially etched away with a contrast ratio of $\sim 43$ in etching rate compared with the unmodified regions. Finally, hollow microstructures are formed inside the glass. Since the thermal treatment leaves behind a rough surface, (d) as a last step, the photosensitive glass sample is annealed again to smooth the etched surface.

Further details and a more comprehensive explanation of this method and experimental setup can be found in [14] and references therein.

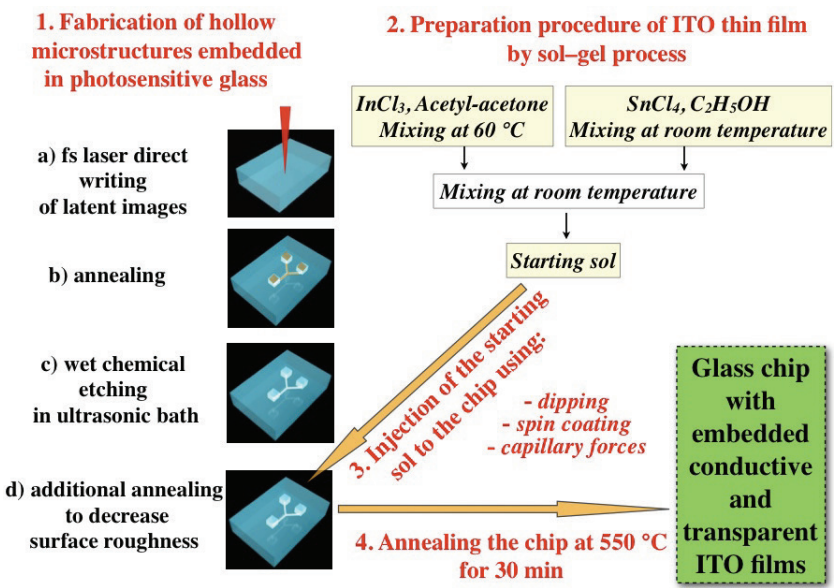

Fig. 1. A four-step experimental method to fabricate ITOcoated 3D structures in photosensitive glass

\section{Results}

The procedure of preparing ITO film can be found in ref. [30], however, in our experiments we annealed the xerogel at $550{ }^{\circ} \mathrm{C}$ for 30 minutes instead of $260{ }^{\circ} \mathrm{C}$ for 10 min. Following preparation of the starting sol, several methods (e.g., using capillary forces, dipping, spin coating) can be used to inject or coat the sol into or on the Foturan chip, respectively. The applied method depends on the level of complexity of the embedded microstructures. In the case of a simple open channel structure, such as a microchannel, a Foturan chip with an embedded channel is placed perpendicularly into the sol. Due to the narrow channel size; the sol is promptly drawn upwards (due to capillary forces) along the wall of the Foturan chip. As a result, a $5 \mathrm{~mm}$ long channel with a $500 \mu \mathrm{m}$ x $500 \mu \mathrm{m}$ crosssection stretching along the Foturan glass chip is obtained (Fig. 2).

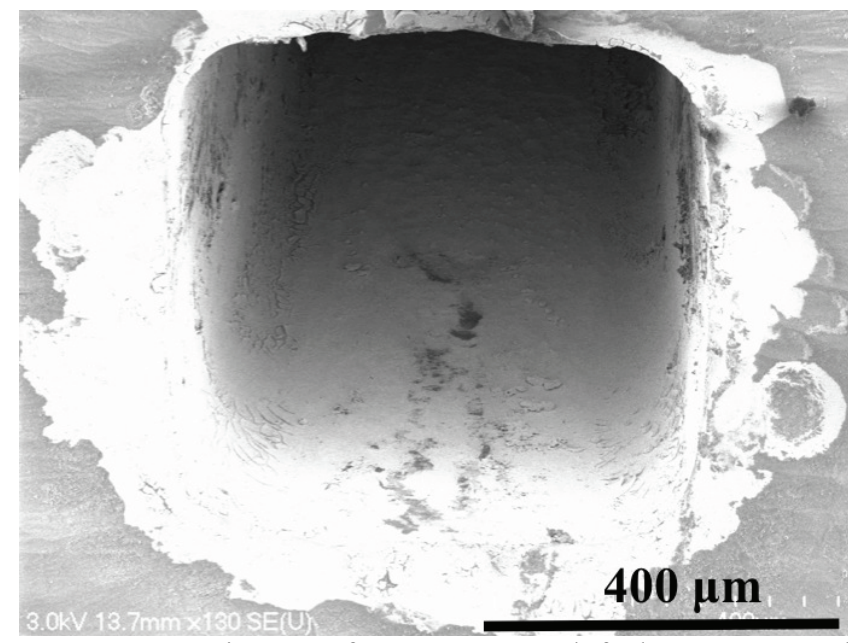

Fig. 2. SEM image of an ITO-coated fs laser-processed microchannel in Foturan glass 
The resulted ITO-coated channels have electrical resistance of $\sim 450 \mathrm{Ohm}$. The resistance was measured between the 2 ends of the channel with a digital resistance meter. Sheet resistivity measurements are impossible to perform due to the narrow and short channel size. The bright area in the SEM image (Fig. 2) shows the thin ITO layer in the channel.

For XRD and optical transmission measurements, the sol was dropped on the Foturan substrate and dried at $50^{\circ} \mathrm{C}$. The XRD data of thick films are presented in Fig. 3. XRD pattern of the dried film, i.e., the as-deposited film, shows only some broadened peaks with low intensity indicating the amorphous structure. Since the as-deposited film is amorphous (Fig. 3a), the Foturan chip was annealed at $550{ }^{\circ} \mathrm{C}$ (for $30 \mathrm{~min}$, then cooled down to room temperature) to form conductive ITO films.

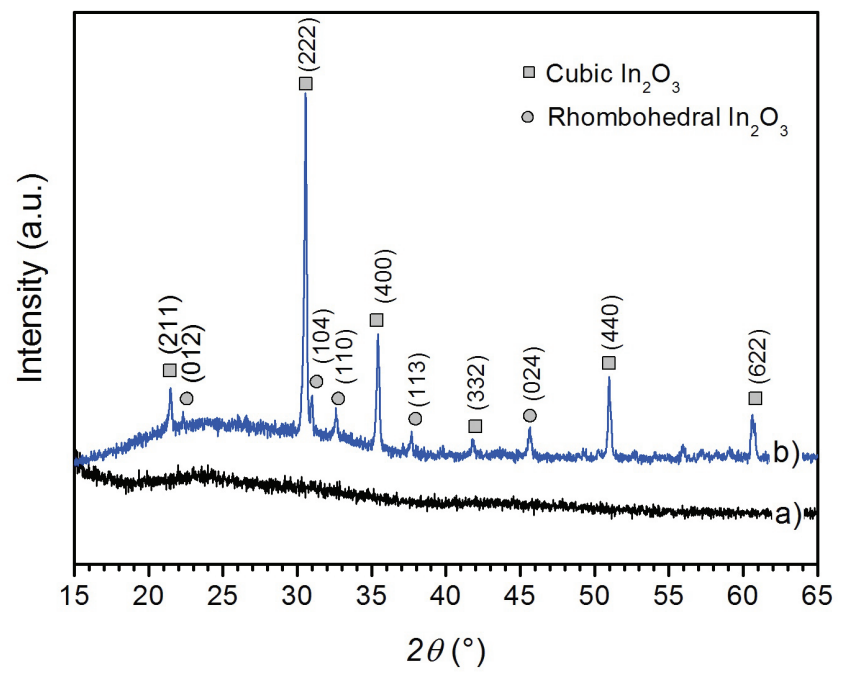

Fig. 3. XRD patterns of (a) dried xerogel film and (b) annealed (crystalline) ITO films [35]

After calcination at $550{ }^{\circ} \mathrm{C}$, sharp reflections appear due to the presence of crystalline, cubic (JCPDS No. 71-2195) and rhombohedral (JCPDS No. 22-0336) indium oxide $\left(\mathrm{In}_{2} \mathrm{O}_{3}\right)$ (see Fig. 3b). The characteristic XRD reflections for the two different crystalline phases (cubic, rhombohedral) are indexed in Fig. 3. It can be seen that between the two phases, the cubic $\mathrm{In}_{2} \mathrm{O}_{3}$ dominates. Tin compounds $\left(\mathrm{SnO}, \mathrm{SnO}_{2}\right)$ were not detected, which confirms the homogenous and successful doping of $\mathrm{In}_{2} \mathrm{O}_{3}$ host by $\mathrm{Sn}^{4+}$ ions.

Figure 4 shows an SEM image of the ITO film on Foturan glass after calcination. The nanocrystalline film homogeneously covers the glass and consists of nanoparticles about $10 \mathrm{~nm}$. Bigger crystallites can be also observed with diameters around $30-50 \mathrm{~nm}$.

Figure 5 presents the optical transmittance of the ITO/Foturan layers and shows that the ITO layer on Foturan glass barely affects the transmittance. The ITOcoated glass is also highly transparent in the visible region.

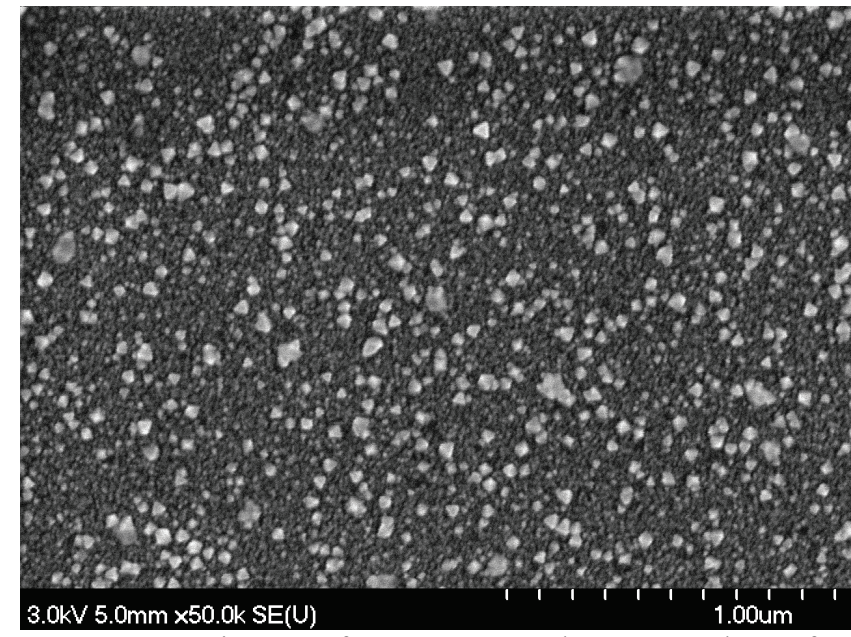

Fig. 4. SEM image of an ITO-coated Foturan glass after calcination

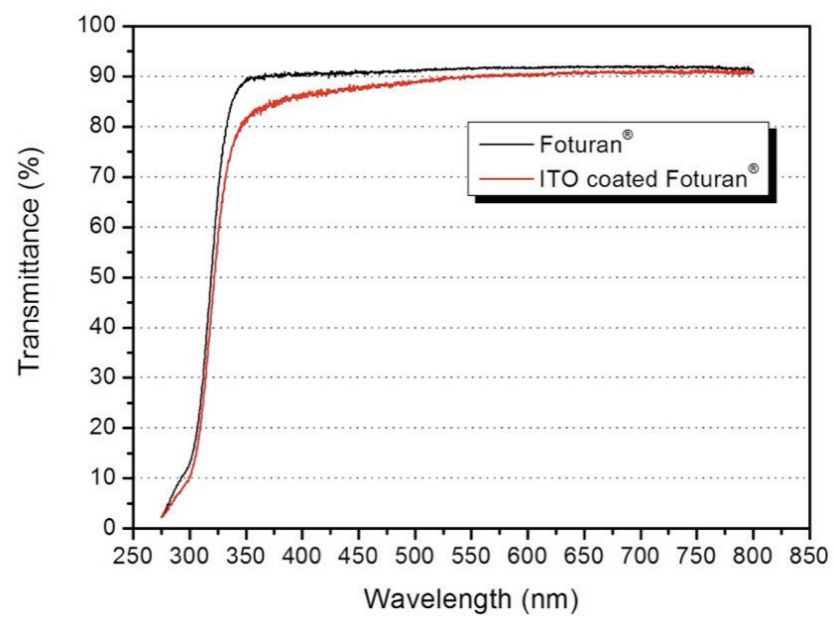

Fig. 5. Optical transmittance spectra of uncoated and ITOcoated Foturan glass

\section{Applications}

When fabricating more complicated 3D structures, a microneedle is used to inject the starting sol into the glass chip. Most of the sol is removed by blowing a highpressure stream of nitrogen into the channel. This process leaves a thin layer of the sol on the inner surface of the 3D microstructure. (A spin coating technique was also exempted, but proved ineffective to remove the sol from the long channel due to a limited rotational speed.) Subsequently, the chip was annealed at $550{ }^{\circ} \mathrm{C}$. As a final processing step, the unwanted ITO-coated portion on the glass surface can be removed by using fs laser ablation.

\subsection{MEMS device}

As we presented in [35], one of the applications of our technique is a transparent MEMS device (Fig. 6). 3D interconnection of electrically conductive lines and reservoirs inside the photosensitive glass was fabricated. Such a 3D interconnection arrangement is desirable to fabricate 
MEMS devices as it allows to applying different voltage between reservoirs. In the presented structure, $2 \times 2$ connecting microchambers (A-C and B-D) are seen where the two connecting microchannels do not intersect (see on the side view of Fig.6). The electrical resistance between the A-C and B-D microreservoirs was found to be $\sim 400$ $\mathrm{Ohm}$, however, it was confirmed that connections between A-B, A-D, C-B, and C-D were completely electrically isolated.

\section{Top view:}

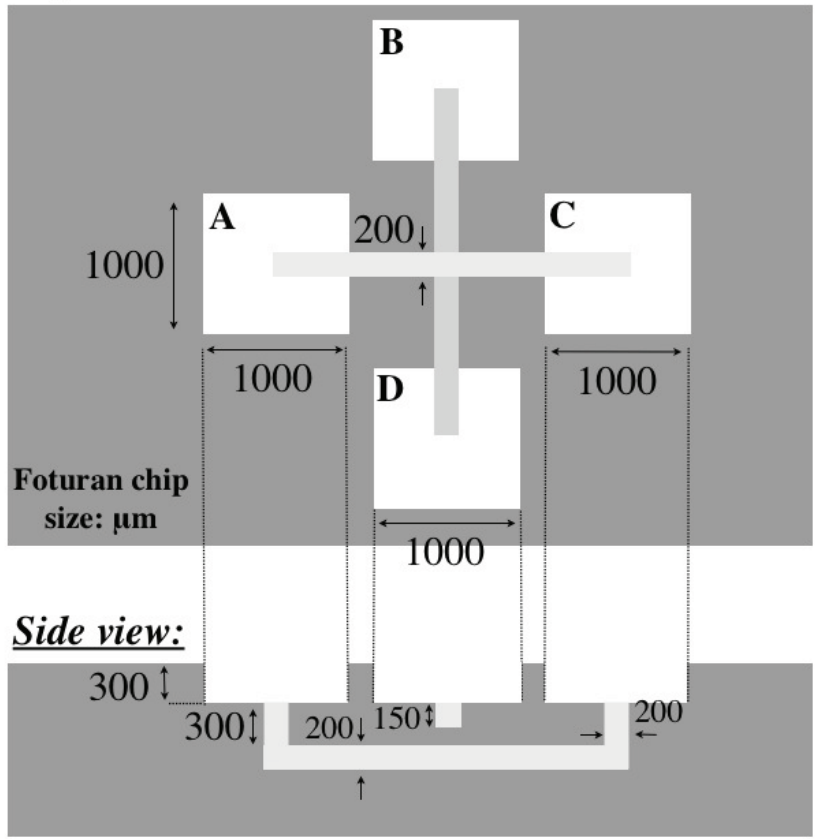

Fig. 6. Microstructure scheme of a possible MEMS layout embedded in Foturan glass [35]

\subsection{Gas sensor}

As for an example of sensor application, Figure 7 presents the concept of a possible $\mathrm{CO}_{2}$ sensor in an electroluminescent cell which can be triggered by $\mathrm{CO}_{2}$ detection. The ITO layers act like plates of a capacitor. Due to the presence of oxidizing gases (e.g. $\mathrm{NO}_{2}, \mathrm{NO}, \mathrm{CO}_{2}, \mathrm{O}_{2}$ ) the conductivity of ITO increases $\left(5 \mathrm{x}\right.$ in case of $\left.\mathrm{CO}_{2}\right)$ [36] producing an electric field in the capacitor that excites the phosphor which emits light. This increase in conductance of ITO can be utilized to initiate the triggering of an electric alarm system. The phosphor on the ITO electrode will only be excited if the ITO has current running through it. The ITO films are transparent, so photons can pass through the layer. The Au layer at the bottom serves as reflecting the generated light to gain more light upon operation. A more detailed explanation of the sensing behavior of ITO can be found in [36].

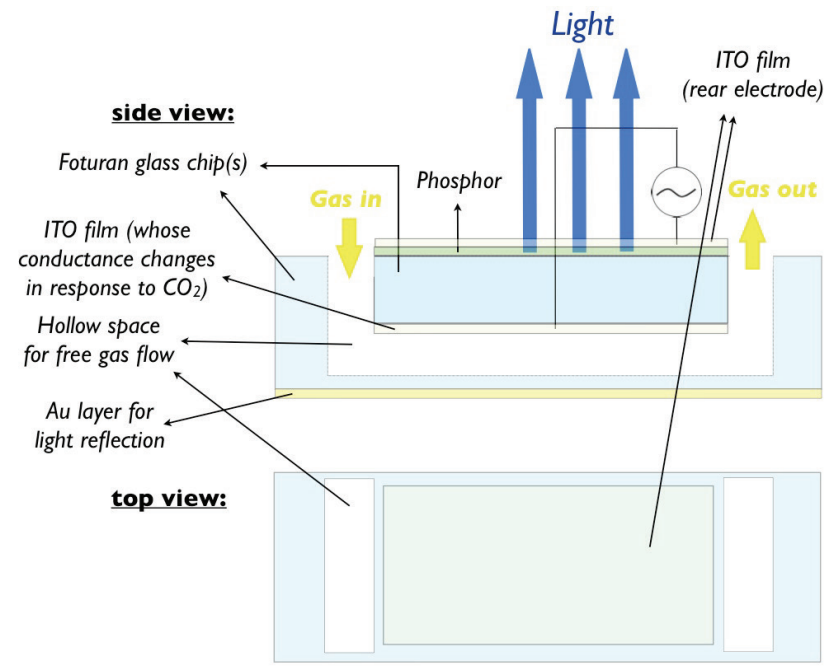

Fig. 7. Concept of a $\mathrm{CO}_{2}$ sensor in an electroluminescent cell

\section{Conclusions}

We have presented a method to fabricate conductive and completely transparent microdevices. The idea is based on the combination of fs laser processing and sol-gel method. This concept will provide a major contribution to strengthen research and development via the realization of a novel microdevice technology. The development of microchips and biochips will promote clinical and medical investigations by this new process and will increase the functionality of the devices and at the same time make them more cost effective for a mass market. Such featured microchips will be highly beneficial for sensitive biochemical analysis and biological, chemical, and medical inspections based on their broad functionality.

\section{References}

1. D. Psaltis, S.R. Quake, C. Yang, Nature 442, 381 (2006) 2. M. Masuda, K. Sugioka, Y. Cheng, N. Aoki, M. Kawachi, K. Shihoyama, K. Toyoda, H. Helvajian, K. Midorikawa, Appl. Phys. A76, 857 (2003

3. Y. Cheng, K. Sugioka, K.Midorikawa, M. Masuda, K.Toyoda, M. Kawachi, K. Shihoyama, Opt. Lett. 28, 55 (2003)

4. Y. Cheng, K. Sugioka, K.Midorikawa, M. Masuda, K. Toyoda, M. Kawachi, K. Shihoyama, Opt. Lett. 28, 1144 (2003)

5. K. Sugioka, M. Masuda, T. Hongo, Y. Cheng, K. Shihoyama, K. Midorikawa, Appl. Phys. A 78, 815 (2004) 6. M. Masuda, K. Sugioka, Y. Cheng, T. Hongo, K. Shihoyama, H. Takai, I. Miyamoto, K. Midorikawa, Appl. Phys. A 78, 1029 (2004)

7. Y. Cheng, K. Sugioka, K. Midorikawa, Opt. Lett. 29, 2007 (2004)

8. K. Sugioka, Y. Cheng, K. Midorikawa, J. Photopolym. Sci. Technol. 17, 397 (2004)

9. K. Sugioka, Y. Cheng, K. Midorikawa, Appl. Phys. A 81, $1(2005)$ 
10. Y. Cheng, K. Sugioka, K. Midorikawa, Appl. Surf. Sci. 248, 172 (2005)

11. T. Hongo, K. Sugioka, H. Niino, Y. Cheng, M. Masuda, I. Miyamoto, H. Takai, K. Midorikawa, J. Appl. Phys. 97, 063517 (2005)

12. Y. Cheng, H. Tsai, K. Sugioka, K. Midorikawa, Appl. Phys. A 85, 11 (2006)

13. K. Sugioka, Y. Cheng, K. Midorikawa, F. Takase, H. Takai, Opt. Lett. 31, 208 (2006)

14. K. Sugioka, Y. Hanada, K. Midorikawa, Laser Photonics Rev. 4, 386 (2010)

15. S. Beke, K Sugioka, K. Midorikawa, A. Peter, L. Nanai, J. Bonse, J. Phys. D: Appl. Phys. 43, 025401 (2010)

16. M. Eritt, C. May, K. Leo, M. Toerker, C. Radehaus, Thin Solid Films 518, 3042 (2010)

17. J.-M. Liu, P.-Y. Lu, W.-K. Weng, Mater. Sci. Eng. B 85, 209 (2001)

18. N.R. Armstrong, C. Carter, C. Donley, A. Simmonds, P. Lee, M. Brumbach, B. Kippelen, B. Domercq, S. Yoo, Thin Solid Films 445, 342 (2003)

19. A.B. Chebotareva, G.G. Untila, T.N. Kost, S. Jorgensen, A.G.Ulyashin, Thin Solid Films 515, 8505 (2007)

20. C.G. Granqvist, A. Hultåker, Thin Solid Films 411, 1 (2002)

21. C.R. Zamarreño, M. Hernaez, I. Del Villar, I.R. Matias, F.J. Arregui, Sens. Actuators B, Chem. 146, 414 (2010)

22. P. Frach, D. Glöß, K. Goedicke, M. Fahland, W-M.
Gnehr, Thin Solid Films 445, 251 (2003)

23. R. Das, K. Adhikary, S. Ray, Appl. Surf. Sci. 253, 6068 (2007)

24. S.-Y. Lien, Thin Solid Films 518, S10 (2010)

25. M. Yamaguchi, A. Ide-Ektessabi, H. Nomura, N. Yasui, Thin Solid Films 115, 447 (2004)

26. N. Manavizadeh, F. Akbari Boroumand, E. Asl-

Soleimani, F. Raissi, S. Bagherzadeh, A. Khodayari, M. Amin Rasouli, Thin Solid Films 517, 2324 (2009)

27. H. Mbarek, M. Saadoun, B. Bessaïs, Mater. Sci. Eng. C 26, 500 (2006)

28. T.L. Li, S.L.C. Hsu, Thin Solid Films 518, 6761 (2010) 29. Y.J. Kang, M. Okada, Y. Haruyama, K. Kanda, S. Matsui et al., J. Photopolym. Sci. Technol. 23, 39 (2010)

30. S. Kundu, P.K. Biswas, Opt. Mater. 31, 429 (2008)

31. E. Celik, U. Aybarc, M.F. Ebeoglugil, I. Birlik, O. Culha, J. Sol-Gel Sci. Technol. 50, 337 (2009)

32. M.J. Alam, D.C. Cameron, Thin Solid Films 455, 377 (2000)

33. T.M. Hammad, Phys. Status Solidi A 206, 2128 (2009)

34. L. Kőrösi, S. Papp, I. Dékány, Thin Solid Films 519, 3113 (2011).

35. S. Beke, L. Kőrösi, K. Sugioka, K. Midorikawa, L.

Dékány, Appl. Phys. A 102, 265 (2011)

36. N.G. Patel, K.K. Makhija, C.J. Panchal, Sensor Actuat B-Chem, 21, 193 (1994)

(Received: June 16, 2011, Accepted: December 19, 2011) 